\title{
Ueber die Geschmacksorgane der Säugethiere und des Menschen.
}

Von

\author{
Dr. G. Schwalbe.
}

Hierzu Taf. XII u. XIII.

In einer vorläufigen Mittheilung im vorigen Bande dieses Archivs „über das Epithel der Papillae vallatae" habe ich eigenthümliche Gebilde im Epithel der Seitenwände jener Papillen beschrieben, die man nach Allem für die Endorgane der Geschmacksnerven bei den Säugethieren halten muss. Sie wurden dort wegen der ihnen zugeschriebenen Function und wegen ihrer grossen Aehnlichkeit mit den „becherförmigen Organen" der Fische als "Sch meckbecher" bezeichnet. Sehr erfreulich ist es, dass diese Beobachtungen zu derselben Zeit, als meine vorläufige Mittheilung publicirt wurde, eine auffallende Bestätigung erhielten, indem Chr. Lovén in Stockholm in einer Arbeit: „Bidrag till kännedomen om tungans smakpapiller,“ die sodann auch in deutscher Sprache und zwar im vorigen Hefte dieses Archirs erschien, im Wesentlichen zu ganz denselben Resultaten gekommen ist. Ich habe mich seitdem anhaltend und eingehend mit diesem Gegenstande weiter beschäftigt. Meine Beobachtungen betreffen jetzt die Zunge vom Schaf, Rind, Reh, Schwein, Pferd, Katze, Hund, Kaninchen, Hase und Meerschweinchen. Ueberdies hatte ich das Glück, menschliche Zungen schon zwei bis fünf Stunden nach dem Tode, also in hinreichend frischem $\mathrm{Zu}$ stande, in genügender Zahl untersuchen zu können. 
Die eben genannten Säugethiere lassen sich je nach der Zahl und Anordnung ihrer immer nur auf dem hinteren Theile der Zunge befindlichen Papillae vallatae in drei Abtheilungen scheiden. Zur ersten gehören die Wiederkäuer: Schaf, Rind und Reh. Bei diesen ist das Vorkommen der Wallpapillen auf zwei seitlich vom hinteren Theile des Zungenrückens gelegene längliche Stellen beschränkt, innerhalb welcher je zehn bis fünfzehn Papillen.von verschiedener Grösse und in verschiedenem Abstande von einander ihren Platz finden.

Anders verhält sich die Zunge der zweiten Gruppe, zu welcher wir Pferd, Schwein und die Nagethiere (Hase, Kaninchen, Meerschweinchen) rechnen müssen. Bei allen diesen Thieren finden sich nur zwei Papillen von gleicher Grösse, auf jeder Zungenhälfte eine, und zwar ungefähr an der Stelle, welche beim Menschen Papillae vallatae trägt. Dieselben zeigen meist einen beträchtlichen Durchmesser. Sehr gross sind sie beim Pferd, wo sie fast einen halben Zoll im Durchmesser erreichen und eine manuigfach zerklüftete Oberfläche, so wie einen nur mangelhaft entwickelten Ringwall erkennen lassen. Zuweilen findet sich etwas hinter diesen beiden Papillen in der Medianlinie der Zunge, also genau an der Stelle, welche dem Foramen coecum der menschlichen Zunge entspricht, noch eine kleinere, die ebenfalls eine zerklüftete Oberfläche und niedrigen Wall besitzt. Die beiden Geschmackswarzen des Schweines dagegen zeigen einen Ringwulst, der vollkommen die Höhe der von ihm eingeschlossenen breiten und oben abgeplatteten, aber mit kleinen Furchen versehenen Papille erreicht, während die der Nager klein sind und nichts Besonderes darbieten.

Zur dritten Gruppe endlich gehört die Zunge der Raubthiere (Hund, Katze) und die des Menschen. Hier finden sich immer mehr als zwei Papillen, beim Hund und bei der Katze jederseits gewöhnlich drei (bei letzterer manchmal auch nur zwei auf jeder Seite der Zunge); beim Menschen ist bekanntlich die gewöhnliche Zahl auf der ganzen Zunge sieben bis neun. Das Gemeinsame der menschlichen und Raubthier-Zunge liegt also weniger in der Anzahl als in der Anordnung der Geschmackspapillen. Letztere bilden nämlich bei allen zur dritten Gruppe gehörigen Thieren einen nach hinten spitzen Winkel, in dessen Scheitel beim Menschen das Foramen coecum sich befindet, das aber auch durch eine Papille ausgefüllt sein kann. Bei Hund und Katze habe ich dagegen nie ein Foramen 
coecum gefunden, aber auch hier bilden die drei Papillen der linken Zungenhälfte mit den dreien der rechten einen nach vorn offenen Winkel. Was dieselben ferner von denen der zweiten Abtheilung unterscheidet, ist, dass sie meist von ungleicher Grösse sind, wie dies Verhalten ja beim Menschen sehr deutlich ausgesprochen ist. Beim Hund und der Katze sind meist die entsprechenden Papillen verschiedener Hälften einander gleich, während die derselben Zungenhälfte unter sich ungleich sind. Beim Hunde kommen ferner dadurch Unregelmässigkeiten zu Stande, dass zuweilen eine Papille auf einer Seite verkümmert, so dass auf dieser Seite sich nur deren zwei vorfinden.

In Betreff der ausserorentlich grossen Schwankungen, welche die Papillae vallatae in ihrer Form und in der Entwickelung des Ringwalls erleiden, kann ich für die des Menschen auf $\mathrm{H}$ enle verweisen, der diese Verhältnisse genau beschreibt ${ }^{1}$ ). Diese grossen Verschiedenheiten in der Gestalt kommen aber nicht den menschlichen Geschmackswärzchen allein zu. Auch bei den Wiederkäuern findet man zahlreiche Abweichungen von der gewöhnlichen Form. Die gewöhnlichsten derselben erwähnt Lovén beim Kalbe; ich brauche deshalb auf diese nicht noch einmal einzugehen. Gedenken muss ich jedoch einer interessanten Complication der Gestalt der umwallten Papillen beim Reh (Fig. 5). Es kommt nämlich hier vor, dass auf die obere Fläche einer regelmässig gebildeten Papilla vallata gleichsam noch eine fungiformis von kleinerem Durchmesser aufgesetzt ist, die also ihrerseits durch keinen Wall mehr geschützt wird. Andererseits findet man beim Ochsen Papillen, von denen nur ein kleines warzenförmiges Stück der Oberfläche sich frei der Mundhöhle zuwendet, während der grösste Theil durch den mächtig entwickelten und verdickten, stark überhängenden Ringwall geschützt wird (Fig. 7). Bei den Wiederkäuern und beim Schweine ist ferner der centrale Theil der der Mundhöhle zugewendeten Papillenseite oft dellenförmig eingedrückt (Fig. 6). Beim Hunde finden sich nicht selten mehrere solcher Eindrücke auf einem Geschmackswärzchen. Auch beim Menschen kommen Findrücke der genannten Stellen vor. Ferner sieht man öfter schon mit unbewaffnetem Auge auf der Oberfläche menschlicher Papillae vallatae wirkliche feine P oren. Es entsprechen dieselben den Ausführungsgän-

1) Eingeweidelehre p. 126. 
gen acinöser Drüsen, welche das bindegewebige Stroma und Oberflächenepithel mancher umwallter Papillen durchsetzen. An einem Durchschnitt, senkrecht auf die Oberfläche der Zunge durch eine solche Papille geführt, überzeugt man sich schon bei schwacher Vergrösserung leicht von diesem Verhalten (Fig. 4).

Das Vorkommen acinöser Drüsen im Bereich der Papillae vallatae ist nun aber nicht etwa auf diese Fälle beschränkt; es finden sich deren vielmehr ohne Ausnahme bei allen von mir untersuchten Säugethieren. Nur ist in dem beschriebenen Falle die Ausmündungsstelle eine abnorme. Gewöhnlich münden sie in den Wallgraben und zwar in den meisten Fällen am Grunde desselben in ziemlich reichlicher Zahl. Die Ausführungsgänge zeigen ein regelmässiges einschichtiges Cylinderepithel und können auch weiter oben am Walle, aber immer im Bereich des Ringthals ausmünden, wie beim Ochsen (Fig. 7), beim Schwein (Fig. 6) und beim Menschen. Die eigentliche Drüsenmasse zeigt auf dem Flächenschnitt einen kreisförmigen Contour und erstreckt sich seitlich um ein bis anderthalb Papillendurchmesser über den Umriss der umwallten Papille hinaus. Letztere sind also an ihrer Basis von einem regelmässigen Kranze acinöser Drüschen umgeben, deren Umfang und Anordnung man schon ohne Mikroskop erkennt. Interessant war es mir, zu constatiren, dass während eine jede Papilla circumvallata von vielen der beschriebenen Drüschen umkränzt wird, solche in der Umgebung echter Papillae fungiformes gänzlich fehlen.

Ein weiterer Unterschied zwischen diesen beiden Papillenarten besteht in der Art der Vertheilung der secundären Papillen. Während dieselben bei den pilzförmigen über die ganze Obertäche verbreitet sind, finden wir sie bei den umwallten nur auf der freien der Mundhöhle zugekehrten Seite. An den Seitenflächen dagegen fehlen sie gänzlich, soweit dieselben einen capillaren Wallgraben begrenzen und durch einen Ringwall geschützt sind. Da es nun aber unvollständig umwallte Papillen giebt, so dürfen wir erwarten, an den freien Seitenflächen auch secundäre Papillen zu finden; und dem ist in der That so. Instructiv ist in dieser Beziehung ein schon oben erwähntes Präparat vom Reh (Fig. 5). Hier besitzt der untere umwallte Theil keine secundären Papillen, während der obere abgeschnürte Knopf deren aufweist (vgl. ferner Fig. 4 vom Menschen).

Begreiflicher Weise ist diese Verschiedenheit in der Vertheilung der secundären Papillen auf einem Geschmackswärzchen von 
wesentlichem Einfluss auf die Configuration des Epithels. Während dasselbe an den Seitenwänden auf dem senkrechten Schnitt beiderseits durch eine gerade Linie begrenzt wird, ist der innere Contour des Oberflächenepithels durch die eindringenden secundären Papillen tief eingekerbt; der äussere Contour dagegen zieht. glatt über die Spitzen der letzteren hinweg, und zwar pflegt im Allgemeinen zwischen den Spitzen der secundären Papillen und der Oberfläche des Epithels noch ein Zwischenraum von 0,1 Mm. zu bleiben. Auf Flächenschnitten durch eine umwallte Papille erscheint dagegen die innere Begrenzung des Epithels gegen das Bindegewebe leicht zackig (Fig. 8). Man muss diese Zeichnung ableiten von senkrecht geschnittenen niedrigen Bindegewebsleisten, die auf der Oberfläche des bindegewebigen Stromạ's senkrecht oder schief zur Zungenfläche verlaufen.

Wenden wir nun unsere Aufmerksamkeit dem Epithel selbst näher zu, so fällt zunächst auf, dass es auf der ganzen Oberfläche eines Geschmackswärzchens von weit geringerer Mächtigkeit ist, als auf anderen Theilen der Zunge. Besonders auffallend ist der Dickenunterschied bei Vergleichung mit dem sehr verdickten Epithel der äusseren Fläche des Ringwalls. Bei manchen Thieren ist diese Verdickung schon makroskopisch zu erkennen. So findet sich z. B. beim Ochsen um jede Papilla vallata herum ein grauweisser Ring, entsprechend dein verdickten Wallepithel (vgl. Fig. 7).

Wenn man ferner die Dicke des Epithels der freien Oberfläche und des Papillen-Abhangs mit einander vergleicht, so stellt sich heraus, dass letzteres im Allgemeinen eine dünnere Lage bildet und zuweilen selbst von geringerer Mächtigkeit ist, als der kleinste Abstand zwischen Spitze der secundären Papillen und freier OberHäche. Dies Verhalten ist besonders auffallend an senkrechten Schnitten durch die Papillae vallatae des Schafes, wo das Epithel der Wallseite $0,08 \mathrm{Mm}$. dick ist, während der Abstand zwischen Spitze der secundären Papillen und Oberfläche meist $0,11 \mathrm{Mm}$. beträgt. Bei anderen Thieren ist dieser Dickenunterschied nicht so deutlich und kann hier auch das umgekehrte Verhältniss eintreten. Es kommt hierbei offenbar sehr auf die Richtung der Schnitte an. An Schnitten, die nicht durch den Mittelpunct der Papille gehen und besonders an solchen, die nicht ganz senkrecht zu ihrer Oberfläche geführt sind, wird man das Epithel der dem Walle zugekehrten Seite meist dicker erhalten, als dem wirklichen Verhalten entspricht. 
Wie auf der ganzen Zungenoberfläche, so kommt auch auf den Papillis vallatis, wenn auch in viel geringerer Mächtigkeit, eine Schicht stark abgeplatteter hornartiger, aber noch kernführender Epithelzellen vor. Diese Schicht ist am dicksten auf der freien Oberfläche der Papille, um auf die Seiten übergehend nach dem Grunde des Ringthals zu allmählich zu verschwinden. Zuweilen scheinen an diesen Zellen noch Kerntheilungen vorzukommen. Wenigstens deutet wohl der biscuitförmig eingeschnürte Kern der Zelle Fig. 17 e auf einen analogen Process.

Die oben beschriebene Schicht von Epithelialplättchen, die man als Hornschicht bezeichnen kann, setzt sich meist ziemlich scharf von den darunter gelegenen Straten vollsaftiger Pflasterpithelien ab. Letztere erscheinen hier meist als Stachel- oder Bürstenzellen, die nichts Erwähnenswerthes darbieten. Dagegen finden sich an den dem Walle zugekehrten Seiten der Papillae vallatae eigenthümliche kleine Bürstenzellen mit relativ sehr grossem Kern und kleinem Zellkörper, welch' letzterer oft an einem Ende in eine lange Spitze ausgezogen ist (Fig. 17 a und b).

Auf andere Weise modificirt ist die unterste Zellschicht, welche unmittelbar dem Bindegewebe aufsitzt. Sie besteht aus eigenthümlichen cylindrischen Zellen, die in ihrem der Peripherie zugewandten abgerundeten Kopfe den Zellkern bergen, während die dem Bindegewebe zugekehrte Basis in feine Spitzen und Zacken ausläuft, die einen innigen Zusammenhang mit dem Stroma zu vermitteln scheinen (Fig. $17 \mathrm{c}$ und d, Fig. 14, 21). Dafür spricht auch der Umstand, dass diese Zellschicht an Präparaten, welche durch Maceration in Lösungen von Kali bichromicum gewonnen sind, oft fest auf der bindegewebigen Unterlage haften bleibt, während alle anderen Zellen abfallen. Wir wollen sie als Basalzellen bezeichnen. Sie sind besonders charakteristisch an den Seitenwänden der Geschmackswärzchen, während sie sich auf und zwischen den secundären $\mathrm{Pa}$ pillen kaum noch von den anderen Zellen unterscheiden.

Es sei mir hier gestattet, noch eines Befundes zu gedenken, der bei der Untersuchung verschiedener Schafzungen sich mir nicht selten ergeben hat. Es ist dies das Vorkommen verästelter Pigmentzellen innerhalb der tiefen Schichten des Epithels der Papillae vallatae und fungiformes. Schon makroskopisch zeichnen sich solche Zungen durch eine dunklere Färbung aus; besonders treten die umwallten sowohl, als pilzförmigen Papillen als schwarze Warzen 
hervor. Macht man nun senkrechte Schnitte durch eine solche schwarze Papille, so erkennt man alsbald schon bei schwacher Vergrösserung, dass in den tiefsten Lagen des Epithels zahlreiche schwarze grobkörnige Pigmentzellen liegen, die nach den verschiedensten Richtungen hin lange Ausläufer mit eben solchen Pigmentkörnchen senden. Besonders ausgezeichnet sind die schwarzen Fäden, welche vom Zellkörper aus unter zeitweiliger Abgabe von Seitenästchen und stellenweisen Knickungen gerade zur Oberfläche hinstreben und auf diese Weise oft zwei Dritttheile des ganzen Epithels durchsetzen. Fig. 2 A and B erläutern die betreffenden Verhältnisse. Fig. A ist nach einer Zeichnung des Herrn Prof. Max Schultze gestochen, der schon früher dieselbe Beobachtung gemacht hatte und so gütig war, mir die Zeichnung zur Publication zu übergeben. Der Schnitt ist einer Papilla fungiformis entnommen, während die ähnliche von mir angefertigte Zeichnung Fig. 2 B einem Präparate von einer Papilla vallata entnornmen ist. Max Schultze theilte mir mit, dass er schon bei Gelegenheit seiner Untersuchungen über die Geruchsschleimhaut beobachtet habe, wie eine Pigmentirung in dieser zuweilen von einer ähnlichen in der Zunge begleitet werde. Die Pigmentzellen finden sich nun nicht etwa bloss zwischen den secundären Papillen, sondern auch im Epithel der Seitenwände zwischen den unten zu beschreibenden Schmeckbechern. Erwähnt muss noch werden, dass ausser den Ausläufern, die mit dem Zellkörper, welcher gewöhnlich einen bellen Kern durchscheinen lässt, zusammenhängen, noch kleine Fäden und Haufen von Pigmentkörnchen zwischen den tiefsten Epithelzellen vorkommen, die nicht mit einer Pigmentzelle in Continuität.stehen, wie dies besonders Fig. $2 \mathrm{~A}$ erkennen lässt. Offenbar sind dies jedoch nur abgelöste Zellfortsätze, und hat man den Grund zu dieser Lostrennung am Wahrscheinlichsten in der Contractitität der farblosen Grundsubstanz unserer verästelten Zellen zu suchen, die ich freilich nicht direct beobachtet habe ${ }^{1}$.

1) Die beschriebenen Pigmentzellen gleichen also ganz denen, welche nach den Untersuchungen ven Max Schultze in der obersten Bindegewebsschicht einer schwarz gefärbten Regio olfactoria sich vorfinden. Vergl. M. Schultze, Untersuchungen über den Bau der Nasenschleimhaut. Halle 1862. p. 56 u. 57. 
Wenden wir uns nun wieder zum Epithel der gewöhnlichen umwallten Papillen, so sehen wir, dass dasselbe überall da, wo es durch einen Wall beschirmt wird, wo keine secundären Papillen stehen, in seiner ganzen Dicke von eigenthümlichen knospenförmigen Gebilden durchsetzt wird, die ich in meiner ersten Mittheilung Schmeckbecher gerannt habe. Lovén bezeichnet dieselben als "Geschmacksknospen, " und werde ich mich in der Folge abwechselnd beider Namen bedienen.

Zur ersten Orientirung sind hier am geeignetsten senkrechte Schnitte durch die Papillae vallatae, nachdem man dieselben zuvor in Ueberosmiumsäure von ein Procent erhärtet hat. Kleine dünneZungenstückchen werden durch dies mit Recht von Max Schultze so gerühmte Reagens so vorzüglich und gleichmässig und dabei mit Erhaltung der feinsten Structurverhältnisse erhärtet, wie dies durch keine andere Flüssigkeit in gleichem Maasse erzielt wird. Nur muss man sich hüten, zu dicke Stücke einzulegen, da die Ueberosmiumsäure bekanntlich nicht tief in die Gewebe hineindringt. Sodann rathe ich die Präparate nicht länger als 24 Stunden in der Flüssigkeit liegen zu lassen und dann gleich Schnitte anzufertigen, die man zweckmässig durch Glycerin durchsichtiger macht. Später werden auch bei Aufbewahrung in starkem Alkohol die eingelegten Stücke brüchig, und, was besonders die Anfertigung feiner Schnitte hindert, sie dunkeln sehr nach, so dass sie schon nach wenigen Tagen eine kohlschwarze Farbe angenommen haben. Die gleich angefertigten Schnitte dagegen sind braun gefärbt und werden, in Glycerin eingelegt, kaum dunkler.

Andere Erhärtungsmethoden leisten hier nicht viel, am wenigsten die Erhärtung in Alkohol, da dieser gerade die Theile, worauf es ankommt, schrumpfen macht, so dass man sie von dem gewöhnlichen Epithel nicht gut unterscheiden kann. Etwas zweckmässiger ist schon die Erhärtung in Kali bichromicum von drei Procent oder in Holzessig; jedoch erreichen die betreffenden Schnitte nicht die Klarheit, wie Ueberosmiumsäure-Präparate.

Betrachtet man nun an letzteren das dem Ringwall zugekehrte Epithel, welches durch Ueberosmiumsäure eine braune Farbe angenommen hat, genauer, so findet man in ihm längs der ganzen Wallseite knospenförmige Gebilde, die sich durch ihr helles Aussehen deutlich ron dem dunkler gefärbten Epithel abgrenzen. Sie erscheinen bei oberflächlicher Betrachtung als Lücken in demselben; und in der 
That sieht man an vielen Stellen wirklich nur Epithellücken, da bei der angegebenen Behandlung die fraglichen Körper leicht herausfallen. Man überzeugt sich ferner leicht, dass diese hellen Knospen das Seitenepithel in seiner ganzen Dicke vom bindegewebigen Stroma an bis zu der das Ringthal begrenzenden Oberfläche durchsetzen (vgl. Fig. 1, 3 bis 7 ; Fig. 21).

Wenn man nun auch im Allgemeinen schon an gut gelungenen Schnitten die Gestalt dieser Gebilde erkennen kann, so ist es doch vortheilhafter, dieselbe an isolirten Objecten zu studiren, und zu diesem ' weck empfiehlt sich wiederum eine höchstens 24stündige Behandlung der Papillen mit Ueberosmiumsäure-Lösung von ein halb bis ein Procent. Will man dünnere anwenden, so ist es nöthig, die Papillen isolirt hineinzulegen, weil schwache Solutionen der Säure schwer bis in den capillaren Wallgraben hinein ihre Wirkung äussern können, indem sie schon vorher vollständig reducirt werden. Uebrigens habe ich keinen Vortheil durch Anwendung $1 / 8^{-}$bis $1 / 16$-procentiger Ueberosmiumsäure erhalten. Schneidet man nun ein Stückchen der Seitenwand einer so behandelten Papilla vallata $a b$ und zerzupft es fein auf dem Objectträger unter Zusatz von Wasser, so erhält man mit Leichtigkeit zahlreiche isolirte Schmeckbecher und gewinnt einen klaren Ueberblick über ihre Gestalt. Es sind dies, wie ich schon in meiner früheren Mittheilung angeführt habe, im Allgemeinen knospenförmige Gebilde. Sie sitzen mit ziemlich breiter kreisrunder Basis dem Bindegewebe auf, sind also anfangs cylindrisch, um sich dann nach der Peripherie zu allmählich zu verbreitern, ihre grösste Dicke nicht weit unter der äusseren Obertäche $z u$ erreichen und sich dann schneller, als sie an Dicke zunehmen, wieder zu verschmälern und gleichsam knospenförmig zu schliessen (vgl. Fig. 1; besonders Fig. 11 und 21). Sie sitzen also breit dem Bindegewebe auf und enden spitz an der Oberfäche des Epithels. Dass ihre natürliche Lage nicht etwa umgekehrt ist, lehren die Schnittpräparate (Fig. 1 und 21).

Die eben gegebene Beschreibung wurde wesentlich nach den beim Schaf gefundenen Verhältnissen entworfen. Es passt dieselbe aber der Hauptsache nach auf die Gestalt der Schmeckbecher aller von mir untersuchten Säugethiere. Es bestehen hier nur geringe Verschiedenheiten, die sich besonders auf die bald mehr peripherische, bald mehr centrale (basale) Lage des grössten Dickendurchmessers und auf die bald schlanke, bald plumpe Configuration derselben 
beziehen. Bei den schlankeren Formen liegt die dickste Stelle des Schmeckbechers meist weiter von der Peripherie ab, als bei den dicken gedrungenen. $\mathrm{Zu}$ den schlanksten gehören die betreffenden Gebilde des Schweines und Ochsen (Fig. 6 und 7). Bei den meisten anderen und beim Menschen finden sich mittlere Verhältnisse, während mir die Schmeckbecher des Rehs besonders gedrungen erschienen sind. Hier verhält sich nämlich der grösste Dickendurchmesser zum Längendurchmesser wie fünf zu sieben, beim Menschen wie eins zu zwei, beim Schwein dagegen wie fünf $z \mathbf{u}$ dreizehn. Die schlanksten Schmeckbecher sind zugleich die längsten mir bekannten und erreichen z. B. die des Ochsen die Länge von $0,172 \mathrm{Mm}$., während die des Menschen zwischen 0,077 und 0,081 Mm. schwanken. Die kleinsten Geschmacksknospen finden sich beim Hund, Reh und Hasen. Bei allen dreien beträgt die Länge im Durchschnitt $0,072 \mathrm{Mm} .{ }^{1}$ ).

An isolirten Schmeckbechern erkennt man ferner, dass aus der Basis derselben viele feine, zum Theil knopfförmig angeschwollene Fäserchen hervorstehen. Ich werde auf letztere bei der Betrachtung der feineren Zusammensetzung der Schmeckbecher zurückkommen. Sodann bemerkt man an den in unversehrtem Zustande isolirten Gebilden eine Streifung, von der Spitze zur Basis ziehend. Die Streifen convergiren nach der Spitze zu (Fig. 11). Auch zahlreiche elliptische Kerne, mit ihrem Längsdurchmesser parallel dem Längsdurchmesser des Schmeckbechers gestellt, zeigen sich jetzt schon deutlich. Es hält jedoch schwer, die dazu gehörigen Zellenkörper abzugrenzen, da dieselben $\mathrm{zu}$ einer compacten Masse verschmolzen erscheinen, deren feinere Zusammensetzung nur noch durch die Längsstreifen und die Kerne angedeutet ist.

1) Der grösste Dickendurchmesser der Schmeckbecher beträgt:

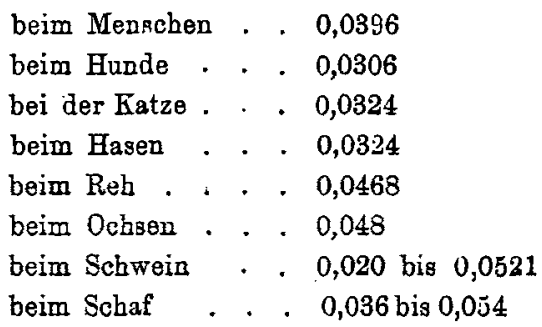

Derselbe ist übrigens keine constante Grösse, und zeigen sich namentlich die Schmeckbecher des Schwoins sebr verschisden dick ( $\mathbf{g l}$. Fig. 3). 
Eine genauere Besprechung verdient die Spitze der Knospen. Schon an Schnittpräparaten erhält man die Gewissheit, dass dieselbe nach dem Ringthale zu frei endigt, von keinen Epithelzellen bedeckt, dass sich über ihr also ein Loch im Epithel befinden muss. An manchen Schnitten durch frisch in Ueberosmiumsäure gelegte Papillen sieht man nun durch dieses Loch hindurch von der Spitze des entsprechenden Schmeckbechers aus ein Bündel feiner Stiftchen über die Oberfläche des Epithels in den Wallgraben hineinragen. Solche Präparate erhielt ich besonders leicht von der Zunge des Ochsen. Man erkennt an ihnen deutlich, dass die Stiftchen aus dem Centrum des Schmeckbechers ihren Ursprung nehmen und gleichsam wie Staubfäden aus einer Knospe hervorragen. Noch besser nimmt man diese Verhältnisse an Geschmacksknospen wahr, die man auf die oben beschriebene Weise isolirt hat. Beim Schafe findet man die Spitze derselben in drei verschiedenen $\mathrm{Zu}$ ständen. Die meisten Schmeckbecher gleichen einer vollständig geschlossenen Knospe, an deren Spitze keine besonderen Structurverhältnisse auffallen. Bei anderen hat sich die Knospe etwas geöffnet, ohne dass man jedoch aus ihr Stiftchen hervorragen sähe. Wohl aber erkennt man rings um den Rand der Oeffnung herum einen Kranz von feinen Härchen, die mit ihren Spitzen convergirend nach innen gerichtet und so gleichsam den Eingang zum Innern der Knospe schirmen (Fig. 13). Bei genauerer Untersuchung sieht man ferner innerhalb dieses Härchenkranzes aus der Tiefe des Schmeckbechers einige feine Stiftchen hervorragen, deren peripherisches Ende sich nicht über das Niveau, in dem die Härchenspitzen liegen, erhebt. Die Stifte entsprechen vollständig den oben erwähnten frei in das Ringthal hineinragenden. Endlich erhält man die Knospen in einem dritten Zustande, der dem vorhin vom Ochsen beschriebenen vollständig entspricht, nämlich mit ganz hervorragenden Stiftchen (Fig. 11 und 12). Letztere können ziemlich weit über die Spitze des Schmeckbechers hervorstehen, nach einigen Messungen bis $0,0072 \mathrm{Mm}$. Man sieht gewöhnlich zwei oder drei neben einander, in seltenen Fällen nur einen. Auch trifft man sie meist von ungleicher Grösse, so dass ein Stiftchen das andere um das Doppelte überragen kann. Sie erscheinen nach Behandlung mit Ueberosmiumsäure als ziemlich starre, glänzende, scharf contourirte Gebilde mit gerade abgestutztem Ende und homogenem Inhalt.

Aus dieser Beschreibung erhellt, dass man die convergirenden, 
den Zugang zum Innern des Schmeckbechers schützenden Hä r ch en wohl zu unterscheiden hat von den aus der Tiefe derselben hervorstehenden Stiftchen, die entweder noch über die Härchen hervorragen können oder auch gänzlich von diesen geschützt zur Beobachtung kommen. Dass in diesem letzteren Falle die äussersten Euden der Stiftchen abgebrochen seien, ist nicht wohl anzunehmen, da sonst schwerlich die Härchen ganz intact geblieben wären. Andererseits zeigen sich die Enden der eingezogenen Stiftchen unter dem Härchenkranz von ganz derselben Beschaffenheit, wie die der hervorgestreckten. Wir müssen also als das Wahrscheinlichste annehmen, dass die Stiftchen bald durch das Loch im Epithel hervortreten, bald sich unter den Härchenkranz zurückziehen können. Durch welchen Mechanismus dies hervorgebracht werde, ob etwa durch Contractilität gewisser unten zu beschreibender zelliegr Elemente des Schmeckbechers selbst, ist für jetzt noch nicht zu entscheiden, ebenso, ob jene beiden Phasen in einer gewissen Beziehung zum Schmeckact stehen.

Von dem Vorhandensein der Härchen und Stiftchen kann man sich auch an ganz frischen Präparaten überzeugen. Sehr geeignet ist dazu eine Flächenansicht der Seitenwand einer Papilla vallata, die man am besten durch Abtragen einer dünnen Lamelle mittelst eines scharfen Rasirmessers erhält. Man bringe dieselbe in verdüntem Jodserum mit ihrer freier Fläche nach oben gewendet und möglichst vor dem Drucke des Deckgläschens geschützt unter das Mikroskop. Bei Einstellung auf die äusserste Oberfläche sieht man dann ohne Anwendung von Reagentien zahlreiche kleine helle, scharf contourirte Kreise in mehr oder.weniger regelmässigem Abstande von einander. Diese Kreise stehen auf dem Gipfel je einer äusserst sanft anschwellenden uhrglasförmigen Erhebung der freien Fläche des Epithels (Fig. 10 a). Ein jeder derselben ist von einem breiten hellen Hofe umgeben, der an frischen Präparaten mit einer gleichfalls kreisförmigen, aber verwischten Begrenzung aufhört. Was uns jedoch an diesen Oberflächenansichten am meisten interessirt, ist der kleine Kreis und seine nächste Umgebung. $\quad$ Er ist der Ausdruck einer scharf begrenzten Oeffnung im Pflasterepithel, welche den Zugang zu je einem Schmeckbecher vermittelt. Die an diese Oeffnung grenzenden abgeplatteten Ptlasterepithezellen zeigen an einem ihrer Ränder einen mehr oder weniger tiefen Ausschnitt, der aber der Form des Loches gemäss immer einem mehr oder weniger grossen Kreisabschnitt entspricht. 
Meist nehmen in dieser Weise drei Zellen an der Begrenzung der Oeffnung Theil (Fig. 10a). Es genügen aber oft zu demselben Zweck schon $z$ wei solcher Zellen, die dann je einen halbkreisförmigen Ausschnitt erkennen lassen (Fig. 10 b). Sehr tiefe Einbuchtungen kommen beim Kalb vor (Fig. $18 \mathrm{a}$ ). Hier genügt aber in vielen Fällen schon e in e Zelle zur Begrenzung einer Oeffnung und zeigt dann dem entsprechend ein scharf contourirtes kreisrundes Loch gewöhnlich nicht weit von einem ihrer Ränder (Fig. 18b). Die an die Schmeckbecher stossenden tieferen Epithelzellen lassen ebenfalls Ausschnitte entsprechend der Form der anstossenden Geschmacksknospe erkennen (Fig. $18 \mathrm{c}$ ).

Wenden wir uns nun nach der Betrachtung dieser Zellformen wieder zu unserer Oberflächenansicht zurück, so sehen wir schon ohne Anwendung von Reagentien, noch besser aber nach Zusatz von dünner Kalilauge, um eine jede Oeffnung herum einen Kranz nach dem Centrum derselben zu convergirender und sich zuspitzender Härchen, die höchst wahrscheinlich identisch sind mit denen, welche oben von isolirten Schmeckbechern beschrieben wurden. Die Härchen sind einigermassen resistent gegen Kalilauge, da sie sich auch nach längerer Einwirkung derselben noch deutlich zeigten. Weniger gut halten sie sich dagegen in Lösungen von Chromsäure oder Kali bichromicum. In letzteren Flüssigkeiten scheinen sie bis auf einen geringen Rest zusammenzuschrumpfen.

Ausser diesen Härchen erkennt man nun bei genauer Betrachtung der kleinen Kreise im Innern derselben vier bis sechs helle stark glänzende Puncte. Um dieselben jedoch scharf zu sehen, muss man den Tubus meist ein wenig tiefer senken, als zur scharfen Wahrnehmung der Härchen nöthig ist. Diese bellen Puncte halten sich genau in ner h a l b der Oeffnungsgrenzen im Epithel. Da ihr Glanz und ihr Durchmesser überdies vollständig mit dem der oben beschriebenen Stiftchen übereinstimmt, so stehe ich nicht an, zu behaupten, dass die betreffenden Puncte als die natürlichen Enden der Stiftchen aufzufassen sind. Dass man an isolirten Präparaten deren meist nur drei, bei Flächenansichten dagegen vier bis sechs wahrnimmt, darf uns nicht wundern, wenn wir bedenken, wie leicht an isolirten Schmeckbechern ein solches Stiftchen abbrechen kann.

Alle diese Verhältnisse sind besonders leicht beim Schaf zu constatiren. Aber auch bei den übrigen von mir untersuchten Säugethieren war es nicht schwer, sich von der Existenz der kleinen 
Löcher im Epithel zu überzeugen. Nicht ganz so befriedigend waren die Ergebnisse in Betreff der Härchen und Stiftchen. Es gelang mir nicht, sie bei allen nachzuweisen. Beim Menschen konnte ich mich wohl von der Existenz der letzteren überzeugen, aber nicht von dem. Vorhandensein eines Härchenkranzes. Die Zahl der Stiftchen ist, nach der Flächenansicht zu urtheilen, hier grösser, als beim Schaf. Es tauchen bei verschiedener Einstellung gegen zehn helle glänzende Puncte auf.

Der Durchmesser der kleinen hellen Kreise ist bei demselben Thiere meist eine constante Grösse. $\mathrm{Er}$ ist beim Schweine sehr klein und misst nur $0,0027 \mathrm{Mm}$; ; beim Hunde beträgt er 0,0045 Mm., beim Schafe $0,0054 \mathrm{Mm}$. Auch beim Menschen schwankt er nur zwischen 0,0027 und $0,0045 \mathrm{Mm}$. Ungleich beträchtlicher sind die Verschiedenheiten beim Kalb, bei welchem äusserst kleine Löcher $(0,002 \mathrm{Mm}$ ) neben relativ grossen vorkommen (von 0,009 $\mathrm{Mm}$. Durchmesser). Dazwischen finden sich aber die allerverschiedensten Maasse.

Soviel über die Bilder, welche man bei Einstellung auf die äusserste Oberfläche des Seitenwand-Epithels erhält. Stellt man nun den Tubus etwas tiefer ein, so verschwinden die kleinen Kreise sammt Härchen und Stiften aus dem Gesichtsfeld; dafür treten aber ihre grossen hellen Höfe, die optischen Querschnitte der Schmeckbecher deutlicher und schärfer hervor. Sie erscheinen jetzt wie scharf begrenzte Löcher in einem dunkeln Maschenwerk, welches durch das gewöhnliche Pflasterepithel der Seitenwand gebildet wird. In Fig. 9 habe ich eine solche Flächenansicht bei tiefer Einstellung abgebildet. Die Umrisse der hellen Schmeckbecher grenzen sich hier scharf von dem dunkeln Epithel ab. Links sind in fünf derselben die kleinen Kreise hineingezeichnet. Noch deutlicher werden diese Verhältnisse an Papillen, die in einer Lösung von doppelt-chromsaurem Kali von zwei bis drei Procent einige Tage lang gelegen haben. An solchen lässt sich oft die Epitheldecke der Seitenwand mit Leichtigkeit vom bindegewebigen Stroma abheben, während die Schmeckbecher meist aus ihren Epithelmaschen herausfallen. Solche Flächenansichten eignen sich auch sehr gut dazu, über die Stellungsverhältnisse der letzteren Klarheit zu erhalten (vergl. Fig. 22). Wo die äussere Fläche der Papille nicht mehr durch den Ringwall geschützt wird, finden sich keine Schmeckbecher melr, andrerseits bildet der Boden des Wallgrabens die untere Grenze der Schmeckbecher- 
zone. Nicht bei allen von mir untersuchten Thieren nehmen nun aber die Geschmacksknospen einen so breiten Ringgürtel ein, wie dies z. B. beim Schaf und Schwein der Fall ist. So findet sich beim Pferde ganz entsprechend dem niedrigen Ringsaum um jede der grossen Papillen nur eine schmale Schmeckbecherzone nahe am Grunde des Wallgrabens, während die ganze übrige zerklüftete Oberfläche zahlreiche sehr lange und dünne secundäre Papillen erkennen lässt. Beim Menschen beschränkt sich das Vorkommen der Geschmacksknospen nur auf die untere Hälfte oder höchstens auf zwei Dritttheile der Seitenwand einer Papilla vallata, sodass das obere Dritttheil fast immer frei von diesen Gebilden ist (Fig. 3 und 4). Andrerseits fehlen hier Schmeckbecher gänzlich an gewissen Stellen der Seitenwand der oben erwähnten unvollständigen Papillae vallatae, an denjenigen Stellen nämlich, welche secundäre Papillen tragen und nicht mehr durch Wall und Graben beschirmt sind (Fig. 4). Dagegen finden sie sich beim Menschen, wenn auch nur vereinzelt, so doch ziemlich constant, an der dem Wallgraben zugekehrten Seite des Ringwalls, welche ein ebenso dünnes Epithel trägt, wie die gegenüberliegende Seite der Papille selbst und keine secundären Papillen erkennen lässt (Fig. 3 und 4). Auch beim Hunde kommen an den entsprechenden Stellen vereinzelte Schmeckbecher vor. Bei den übrigen Säugethieren habe ich deren nur innerhalb der oben näher bezeichneten Ringzone gefunden. Nur beim Schwein bin ich noch mit einer anderen Becher tragenden Stelle bekannt geworden. Bei diesem Thiere findet sich nämlich an jeder Seite der Zunge etwa je einen Zoll lateralwärts von der grossen Geschmackspapille eine glatte mit tiefen unregelmässigen Furchen versehene Stelle auf der Oberfläche der Zunge, ungefähr einen halben Zoll im Durchmesser haltend. Macht man durch dieselbe einen senkrechten Schnitt, so sieht man, dass die meisten dieser tiefen Rinnen von gewöhnlichem Pflasterepithel ausgekleidet sind, das glatt über secundäre Papillen hinwegzieht. In einigen besonders tiefen und engen Spalten lässt dagegen das Epithel einzelne Schmeckbecher erkennen, während zugleich die secundären Papillen fehlen. Bei anderen Thieren war ich nicht so glücklich, ausserhalb des Bereichs der. Geschmackswärzchen Schmeckbecher aufzufinden. Ich habe die Gaumenschleimhaut und den Arcus glossopalatinus vergeblich zu diesem Zwecke durchsucht. Auch an echten Papillae fungiformes gelang es mir nicht, die fraglichen Gebilde 
wahrzunehmen. Ich glaube daher, dass die Angabe Lovén's, dass er in seltenen Fällen auch an pilzförmigen Papillen vom Kalb Schmeckbecher gefunden habe, auf solche $\mathrm{zu}$ beziehen ist, welche Uebergangsformen zwischen den fungiformes und vallatae bilden, wie sie sich fast auf jeder Zunge eines Wiederkäuers finden. Das Vorkommen von secundären Papillen und Schmeckbechern scheint sich immer auszuschliessen. Letztere finden sich nur, wo genügender Schutz gegen äussere Insulte durch einen dicht anliegenden, wohl entwickelten Wall gewährt ist, während secundäre Papillen sich überall da einstellen, wo der.Wall niedrig ist oder weit absteht, also der Graben breit ist.

Betrachten wir nun noch kurz die Vertheilung der Schmeckbecher innerhalb des erwähnten Ringgürtels einer Papilla vallata, so finden wir, dass dieselbe im Allgemeinen eine ziemlich gleichmässige ist (vergl. Fig. 22). Doch lässt sich eine reihenförmige Anordnung dieser Gebilde von dem Gipfel der Papille zum Boden des Wallgrabens bei manchen Thieren, z. B. beim Schwein, nicht verkennen, sodass in dieser Richtung der Abstand zweier Schmeckbecher von einander oft bedeutend geringer ist, als in der darauf senkrechten. Man sieht ferner, dass sie innerhalb ihres Verbreitungsbezirkes sehr dicht neben einander stehen. Am dichtesten stehen sie beim Menschen und nächst diesem beim Hunde, bei ersterem oft so dicht, dass ihre äusseren Grenzen, die bei Oberflächenansicht nach den obigen Auseinandersetzungen als Kreise erscheinen, sich berühren. Durchgehends ist beim Menschen der Zwischenraum zwischen zwei Schmeckbechern kleiner, als ihr grösster Durchmesser. Aehnlich ist es beim Hunde. Bei beiden ist auch die erwähnte reihenweise Anordnung weniger deutlich zu erkennen. Weiter auseinander stehen im Allgemeinen die betreffenden Gebilde der Wiederkäuer und des Schweins, wo der Abstand zweier derselben von einander ein sehr verschiedener sein kann (0,0072-0,036 Mm.).

Um die Gesammtzahl der Schmeckbecher auf einer Papilla vallata und annähernd auf der ganzen Zunge zu bestimmen, zählte ich an senkrecht durch die Mitte einer umwallten Papille geführten Schnitten die Becher, welche in einer Reihe vom Grunde des Wallgrabens bis zur Oberfläche stehen. Ebenso findet man ohne Mühe an Schnitten, parallel der Zungenobertäche geführt, die An- 
zahl derselben Gebilde im Umkreise der Papille. Multiplicirt man dann beide Ziffern mit einander, so hat man offenbar annähernd die Zahl sämmtlicher Schmeckbecher eines Geschmackswärzchens, und diese wieder mit der Zahl der Papillae vallatae multiplicirt, gibt die Gesammtzahl der Schmeckbecher auf der Zunge. Auf diese Weise findet man beim Schaf auf dem Längsschnitt acht, auf dem Flächenschnitt 60 Geschmacksknospen, im Ganzen also deren 480 auf einer Papille mittlerer Grösse. Rechnen wir nun 20 der letzteren auf eine Schafzunge, was eher zu niedrig, als zu hoch angeschlagen ist, so ist die Gesammtzahl der Schneckbecher hier 9600. Eine analoge Berechnung ergibt für das Rind die hohe Summe von $35200 \mathrm{Ge}-$ schmacksknospen, indem hier 22 auf dem Längsschnitt, 80 auf dem Querschnitt gezählt werden und die Anzahl der Papillen durchschnittlich ebenfalls 20 beträgt. Beim Schwein, wo sich nur zwei umwallte Papillen finden, trägt jede derselben allein 4760 , also beide zusammen 9520 Schmeckbecher ${ }^{1}$ ), die ganze Zunge also jedenfalls noch mehr, da sich hier, wie oben erwähnt, deren auch an anderen Stellen finden, wo sich keine ähnliche Schätzung durchführen lässt. Für den Menschen eine gleiche Berechnung vorzunehmen, habe ich für werthlos gehalten, da wegen der so grossen Verschiedenheit der einzelnen Papillae vallatae die an einer derselben gefundenen Zahlen unmöglich für alle Geltung haben können. Man müsste also, um wenigstens für eine Zunge eine Zahl zu finden, die dem wirklichen Verhalten einigermassen entspricht, die Schmeckbecher einer jeden Papille für sich berechnen. Aber die so erhaltenen Resultate würden ebenfalls keinen Anspruch auf allgemeine Gültigkeit machen können, da gerade beim Menschen die Papillae vallatae ausserordentlich grossen individuellen Schwankungen unterworfen sind. Ueberdies macht das Vorkommen vereinzelter Geschmacksknospen am Rĩngwalle selbst eine genaue Berechnung unmöglich.

Um die Schmeckbecher in ihre einzelnen Bestandtheile zu zerlegen, bediente ich mich anfangs sehr dünner Lösungen von Ueberosmiumsäure. Ich habe die verschiedensten Concentrationen von ein achtel bis ein Procent durchprobirt bei verschiedener Dauer der Einwirkung und bin doch zu keinem befriedigenden Resultat damit gekommen. Es gelingt beim Zerzupfen der so behandelten Präparate höchstens,

1) Auf den Längsschnitt kommen 17 , auf den Querschnitt 280 dieser Gobilde. 
einige peripherische Spindelzellen vom Schmeckbecher abzutrennen. Auch mittelst der concentrirten Oxalsäure und nach Anwendung der 35procentigen Kalilauge gelang eine befriedigende Isolirung der Elemente nicht. Die Geschmacksknospen zerfielen zwar leicht in ihre einzelnen Bestandtheile, allein letztere zeigten sich dann meistens so verändert, dass an ein genaues Studium derselben gar nicht zu denken war. Ich sah mich desshalb genöthigt, zum genannten Zwecke Lösungen von Chromsäure oder doppelt-chromsaurem Kali zu benutzen. Erstere, in der Concentration von $1 / 50$ bis $1 / 25 \%$ angewandt, zeigt sich hier nicht besonders vortheilhaft. $\mathrm{Zu}$ einer vollständigen Maceration braucht man damit sehr lange Zeit. Oft sind die eingelegten Stücke noch nach 14 Tagen nicht brauchbar. Wenn endlich das Epithel sich ablöst, erscheinen die Elemente der Schmeckbecher oft so verändert, dass man keinen sicheren Aufschluss über ihre Natur erhäIt. Besser erwiesen sich mir dünne Lösungen von Kali bichromicum $(1 / 4$ bis $1 \%$ ). Sehr oft gelingt es, nach Behandlung mit einer solchen Flüssigkeit gute Präparate zu bekommen; manchmal jedoch lässt die Methode in Stich, ohne dass man den Grund dafür anzugeben weiss: man wartet Wochen lang und schliesslich erhält man keine brauchbaren Präparate, sondern nur massenhaft Pilze. Auch durch häufigen Wechsel der Flüssigkeit lässt sich dieser Uebelstand nicht vermeiden. Ich habe desshalb stärkere Lösungen des doppelt-chromsauren Kali versucht $\left(1 \frac{1}{2}\right.$ bis $2 \%$ ) und dieselben nur wenige Tage einwirken lassen. Der Erfolg war ein weit besserer, als nach Anwendung der dünnen Solutionen, da einmal der Zerfall der Elemente rascher eintrat, andrerseits sich letztere viel weniger verändert zeigten. Noch günstigere Resultate erzielt man in vielen Fällen durch Maceration in Jodserum, dessen ich mich ebenfalls vielfach bediente. Doch verhalten sich auch gegen diese Flüssigkeit die Papillen verschiedener Thiere verschieden; oft erhält man rasch brauchbare Präparate; andererseits tritt in vielen Fällen eher Vibrionenbildung und Fäulniss auf, als Zerfall des Epithels in seine Bestandtheile.

Durch Anwendung der genannten Methoden lassen sich nun die Schmeckbecher, wie ich schon in meiner ersten Mittheilung über diesen Gegenstand erwähnte, in spindelförmige Zellen zerlegen. L ovén, der sich ebenfalls vorzugsweise des Jodserums und dünner Lösungen von Chromsäure und Kali bichromicum bediente, unterscheidet zwei Arten dieser Spindelzellen, die er als Stütz- oder Deck- 
zellen und als Geschmackszellen bezeichnet. Erstere bilden die Hülle der Knospe und schliessen in jedem Schmeckbecher nur ein bis zwei der letzteren ein. Im Wesentlichen stimmen auch hier meine Beobachtungen mit denen Lov én's überein. Auch ich unterscheide die äusseren Zellen mit grösserem Zellkörper und dickerem centralen Fortsatz als Deckzellen von den central gelegenen Geschmackszellen. Eine genauere Untersuchung lehrt Folgendes.

Die Deckzellen (Fig. 14, 15a und b, 16a und b), welche gleichsam die Kelch- und Blumenblätter der Knospe darstellen und sich also an der Peripherie derselben befinden, haben einen im Allgemeinen spindelförmigen Zellkörper mit ovalem Kern obne Kernkörperchen ${ }^{1}$ ). Sie spitzen sich nach der Peripherie, also nach der Spitze des Schmeckbechers hin gewöhnlich feín zu und tragen hier im ganz frischen Zustande wahrscheinlich die feinen Härchen, die oben von Ueberosmiumsäure-Präparaten beschrieben wurden. Mit Sicherheit lässt sich jedoch dies nicht feststellen, da ich an Zellen, die nach Einwirkung von Chromsäure oder doppeltchromsaurem Kali isolirt waren, nie einen haarartigen Aufsatz auf der Spitze gefunden habe. Da nun aber die Härchen im frischen Zustande existiren und, wie wir unten sehen werden, mit den Geschmackszellen Nichts zu thun haben können, so müssen wir sie wohl mit den Deckzellen in Zusammenhang bringen und annehmen, dass sie in den genannten Lösungen so verändert werden, dass sie sich der Beobachtung entziehen. Wahrscheinlich haben wir in den zugespitzten Enden der Deckzellen noch Reste der Härchen vor uns. Der Zellkörper selbst zeigt sich besonders an den äussersten Zellen entsprechend der Configuration des Schmeckbechers leicht bogenförmig gekrümmt, mit der Concavität nach der idealen Achse der Geschmacksknospe gewendet. Der Kern liegt gewöhnlich in der Mitte der spindelförmigen Zelle; jedoch kann er auch der Spitze derselben sehr nahe rücken, beim Schaf z. B. bis auf $0,0036 \mathrm{Mm}$. Hinter dem Kern nach dem bindegewebigen Stroma zu behält nun die Deckzelle anfangs noch ihre durchschnittliche Breite und ihr feinkörniges Aussehen, um sich dann

1) Die Länge des Kerns beträgt beim Menschen 0,0108 Mm., die Breite $0,0072 \mathrm{Mm}$. Bei den Säugetieren schwankt die Länge desselben zwischen 0,0072 und $0,018 \mathrm{Mm}$., die Breite zwischen 0,0072 und 0,008 Mm. Besondere Maasse für die Länge einer Deckzelle zu geben, halte ich für überflüssig, da dieselbe annähernd mit der des betreffenden Schmeckbechers übereinstimmt. 
fast constant in feinere Aeste zu zerspalten, von denen wenigstens einer die bindegewebige Grundlage erreicht, die anderen sich nach weiteren Theilungen zwischen den Ausläufern benachbarter Zellen und den schon oben beschriebenen Basalzellen verlieren. Oft erreichen auch mehrere Fortsätze die Grenze zwischen Epithel und Bindegewebe. Es können demnach die centralen Enden der Deckzellen die mannigfachsten Formen darbieten. Besonders leicht gelang es mir beim Menschen dieselben zu isoliren (Fig. 16 a und b), und sind hier ihre centralen Enden zuweilen knopfförmig angeschwollen. Auch war oft ein inniger Zusammenhang derselben mit dem Bindegewebe zu constatiren, indem ich nicht selten Bilder erhielt, wo die gewöhnlicher Epithelzellen der Seitenwand einer Papille abgefallen waren, nur noch Basalzellen und Schmeckbecher fest auf dem Stroma hafteten. Beim Schaf ist dieser Connex ein wenig inniger; dagegen haften hier die Deckzellen an ihren centralen Enden, welche unter sich dicht verfilzt und verklebt sind, fest an einander; so dass man in den meisten Fällen nur geöffnete Kelche isolirt, aus denen die Schmeckzellen herausgefallen sind (Fig. 14). Auch kommen hier Deckzellen mit unverästeltem centralem Fortsatz vor, wie Fig. $15 \mathrm{a}$ und $\mathrm{b}$ zeigt. Variköse Anschwellungen an den centralen Ausläufern der Deckzellen habe ich bei keinem Thier beobachtet.

Wesentlich anders verhalten sich die Geschmackszellen (Fig. $15 \mathrm{c}$ bis $\mathrm{h}, 16 \mathrm{c}$ bis $\mathrm{f}$ ), die das Centrum eines jeden Schmeckbechers einnehmen. Ihr Kern liegt in der Mitte zwischen peripherischem und centralem Ende, zeigt aber im Wesentlichen dieselbe Beschaffenheit, wie in den Deckzellen. Nur in sehr wenigen Fällen wurde im Innern desselben ein kleines Kernkörperchen beobachtet (Fig. 16 d). Sehen wir von diesen Fällen vorläufig ab, so liegt das Charakteristische der Geschmackszellen darin, dass ihr breiter peripherischer Fortsatz sich allmählig in der Richtung nach dem Epithel zu verschmälert, aber hier nicht spitz mit einem Härchen, sondern abgestutzt endigt, während der centrale Fortsatz schon dicht hinter dem Kerne als dünner glänzender, oft unregelmässige Varicositäten zeigender Faden erscheint, der am centralen Ende gewöhnlich noch eine knopfförmige Anschwellung erkennen lässt. So beschaffen zeigt sich der bei weitem grösste Theil der ziemlich leicht aus dem Innern des Schmeckbechers herausfallenden Geschmackszellen. Der peripherische Fortsatz ist äusserst 
feinkörnig und zart contourirt. An besonders gut conservirten Zellen sieht man jedoch das Ende desselben in ein schmales hellglänzendes, oben scharf abgeschnitten endendes Stiftchen übergehen, das offenbar identisch ist mit denen, welche wir aus den durch Ueberosmiumsäurelösung isolirten Geschmacksknospen hervorragen sahen (Fig. $15 \mathrm{f}$, Fig 16 e). Dass man dasselbe nicht an allen Schmeckzellen wahrnimmt, ist jetenfalls der Einwirkung der Reagentien zuzuschreiben, die besonders auf das peripherische Ende mannigfach verändernd einwirken können. Man betrachte nur die Reihe der in Fig. 15 dargestellten Zellen rom Schaf. Während g eine intacte, noch mit Stiftchenaufsatz versehene Zelle darstellt, zeigt der peripherische Fortsatz anderer unregelmässige Varicositäten (c und e); bel noch anderen ist er schlangenförmig gekrummt und dünn (g), bei anderen (h) kommt er abgebrochen zur Beobachtung. Die erwähnten Varicositäten zeigen sich immer scharf contourirt und glänzend. Was ferner den Kern betrifft, so habe ich ihn nur in wenigen Fällen undeutlich gefunden (Fig. 16 f), und in diesen Fällen zeichnete sich die spindelförmige Anschwellung, in welcher man den Kern vermuthen musste, durch hellen Glanz aus. Vom centralen Fortsatz hebt auch Lovén hervor, dass die Varicositäten äusserst unregelmässig seien. Es kommen auch solche Fortsatze ohne jegliche Varicosität vor, während andere nur wenige, aber dicke erkennen lassen. Das an das bindegewebige Stroma stossende Ende dieses Ausläufers zeigt jedoch fast immer eine knopfförmige oder knollige Anschwellung. Nur einmal, und zwar beim Schafe (Fig. $15 \mathrm{c}$ ), erhielt ich eine Geschmackszelle mit regelmässig varikösem centralen Faden. Lo vén vergleicht die knotigen dickeren Anschwellungen der letzteren, wie sie gewöhnlich vorkommen, mit Myelin-Gerinnseln. Ich kann mich dieser Ansicht nicht anschliessen. Zwar stehen diese Varicositäten und Knollen dem Myelin in ihrem starken Lichtbrechungsvermögen sehr nahe, allein andere Reactionen widersprechen dieser Auffassung, vor Allem der Umstand, dass nach Behandlung mit Ueberosmiumsäure keine blauschwarze Färbung der betreffendenFortsätze, sondern nur eine lichtbraune Farbe eintritt. Ich bin geneigt, die betreffenden Varicositäten von der Gerinnung einer Eiweisssubstanz abzuleiten und mehr der Substanz eines Axencylinders gleich zu setzen. Lovén beschreibt endlich noch am centralen Faden kleine Seitenäste von derselben Beschaffenheit wie der Hauptfaden (siehe Lovén's Figur $3 \mathrm{c}$ und e, 4 b). Ich habe bei einer langen Reihe von 
Untersuchungen dieselben nicht zu Gesicht bekommen, kann mich also nicht über ihre etwaige Bedeutung aussprechen.

Soviel über die gewöhnliche Form der Geschmackszellen. Ich habe schon oben erwähnt, dass in ihnen zuweilen ein Kernkörperchen gefunden wird (Fig. $16 \mathrm{~d}$ ). Diese Zellen zeigen eine etwas andere Beschaffenheit. Ihr peripherischer Fortsatz ist kurzer, gleichmässig breit und vorn abgestutat, ohne Stift. Der centrale Faden dagegen unterscheidet sich kaum von dem der gewöhnlichen Geschmackszellen. Nur ist fast nie eine Andeutung von Varicositäten an ihm zll sehen, wenn man nicht die knopfförmige Endanschwellung als solche bezeichnen will. Doch kann auch diese fehlen, sowie ich auch das Kernkörperchen keineswegs als charakteristisch für diese Art von Zellen ansehe (vergl. Fig. $15 \mathrm{~d}$ ). Vergleicht man dieselben mit den erstbeschriebenen Stiftchenzellen, so wird man wohl kaum daran denken können, sie als durch Einwirkung der Reagentien veränderte Stiftchenzellen aufzufassen, zumal da beide neben einander in demselben Präparat gefunden werden. Man hat also eine gewisse Berechtigung, dieselben als Stabzellen von den Stiftchenzellen zu trennen und beide als $z$ wei verschiedene Arteñ von Geschmackszellen, die möglichenfalls verschiedene Geschmacksempfindungen vermitteln, aufzufassen. Die Stabzellen erinnern sehr an die von A. Ke ${ }^{1}$ ) beschriebenen und abgebildeten Geschmackszellen des Frosches.

Es bleibt nun noch eine wichtige Frage zu beantworten, nämlich die nach der Beziehung der eben als Geschmackszellen beschriebenen Elemente $\mathrm{zu}$ den in das bindegewebige Stroma der umwallten Papillen eintretenden zahlreichen Nervenfasern. Was zunächst das letztere betrifft, so zeigt es eine wesentlich fibrilläre Structur. Die Fibrillenbundel kreuzen sich in den verschiedensten Richtungen und enthalten zahlreiche elastische Fasern. Dem entsprechend finden wir denn auch das Stroma sehr fest und zähe; es lässt sich sehr schwer durch Nadeln fein zerzupfen. Elastische Fasern finden sich in besonders reichlicher Menge beim Pferd, wo auch in jede der sehr langen secundären Papıllen mehrere derselben hinaufsteigen. Von besonders festem und dichtem Gefige ist das Bindegewebe der Papillae

1) Ueber die Endigungsweise der Geschmacksnerven in der Zunge des Frosches. Reichert's und du Bois-Reymond's Archiv 1861. 
vallatae des Schweines, so dass sich durch diese schon an ganz frischen Präparaten leicht dünne Schnitte anfertigen lassen.

Begreiflicher Weise erschwert nun diese zähe Beschaffenheit des Papillenkörpers sehr die Erforschung des feineren Verlaufs der Nervenfasern, während die gröbere Vertheilung derselben schon an Holzessigpräparaten verhältnissmässig leicht zu uberblicken ist. In Betreff der feineren Anordnung der Nervenfasern lässt uns aber die letztere Methode vollständig im Stich. Auch frische feine Schnitte durch Papillae vallatae vom Schwein, durch Glycerin aufgehellt, lassen nicht mehr erkennen. Ich bediente mich auch vielfach der Goldchloridmethode und zwar sowohl der ursprünglich von Cohnheim angegebenen, als der modificirten Gerlach'schen; aber auch durch diese Methode, die für die Cornea so vorzügliche Dienste leistet, erhielt ich hier keine befriedigenden Aufschlüsse. In manchen Fällen zeigten sich allerdings nicht allein die Nervenfasern, sondern auch die Schmeckbecher violett gefärbt, wie dies auch Lovén anfuhrt; allein gerade das Verhalten der Nervenfasern unmittelbar unter jenen blieb unaufgeklärt, obwohl ich, um zu starkes Nachdunkeln zu verhuten, auch sehr dünner Lösungen (bis $1: 1000$ ) mich bediente. In vielen Fällen aber trat die Reaction nach dem Uebertragen der durch Chlorgold gelb gefärbten Schnitte in schwach angesäuertes Wasser gar nicht ein, ohne dass ich einen Grund dafür anzugeben wusste. Setzte ich dagegen einige Tropfen Kreosot hinzu, so erhielt ich zugleich mit der Aufhellung des Präparats lebhafte blauviolette Färbung; fast immer betraf dieselbe aber nicht Nerven und Schmeckbecher, sondern vorzüglich die stärkeren Bindegewebszüge und Gefässe. Manchmal wurde auch das gewöhnliche Epithel der Papillen dunkelblau tingirt; von Nervenfärbung war an solchen Präparaten nichts zu sehen. Interessant ist noch die Thatsache, dass auch an Schnitten, die nach Erhärtung in Ueberosmiumsäurelösung angefertigt sind, zuweilen noch Goldfärbung der schon dunkel gefärbten markhaltigen Nervenfasern eintritt. Letztere nehmen dann ein dunkelviolettes Aussehen an.

Behufs der Erforschung des Verhaltens der Nervenfasern unmittelbar unter den Schmeckbechern sah ich mich somit genöthigt, mich der dunnen Chromsäurelösungen zu bedienen, die für die Geruchsschleimhaut so Vorzügliches leisten. In den umwallten Papillen setzt dagegen die ausserordentliche Zähigkeit des Bindegewebes einem in gleicher Weise befriedigenden Resultat unitberwindliche 
ch wierigkeiten entgegen. Dennoch gewährt auch hier eine mchrtägige Maceration in Chromsaure von $1 / 50 \%$ über Manches Aufschluss, was mittelst anderer Methoden nicht erkannt werden konnte.

Die Anwendung aller genannten Methoden ergibt nun Folgendes. Die Nervenfasern des Glossopharyngeus-Stammes, der sich auch bei den Saugethieren leicht bis in die Gegend der Papillae vallatae verfolgen lässt und sich hier in femnere Aeste fưr die einzelnen $\mathrm{Ge}$ schmackswärzchen theilt, sind zum grössten Theil markhaltig und zeichnen sich durch ihre Feinheit vor anderen markhaltigen Nervenfasern aus. Beim Schaf besitzen sie durchschnittlich eine Dicke von $0,0045 \mathrm{~mm}$.; einige messen nur $0,0036 \mathrm{~mm}$., während andrerseits dickere vorkommen bis zu 0,0062 im Durchmesser. Ausser diesen feinen markhaltigen Fasern habe ich aber schon im Stamm des Glossopharyngeus mit Sicherheit Remak'sche Fasern gefunden, die nach der Peripherie zu zahlreicher zu werden scheinen. In manchen Fällen gelang es mir, den Zusammenhang einiger derselben mit den nach Remak's Entdeckung constant im Stamme des Zungen-Schlundkopfnerven vorkommenden Ganglienzellen nachzuweisen. Beim Schaf findet man bis acht der letzteren zu einem kleinen Ganglion zusammengruppirt; sie sind hier von elliptischer Gestalt, oft durch Druck gegen einander abgeplattet, feinkornig, gelb pigmentirt, mit kugelrundem relativ kleinen Kern und Kernkörperchen. Nach der Peripherie zu scheinen die kleinen Ganglien immer zahlreicher in den Verlauf des Glossopharyngeus-Stammchen sich einzuschieben und oft erst unmittelbar unter den umwallten Papillen aufzuhoren. Wenigstens sah ich beim Schwein noch unmittelbar unter der Basis der grossen Papillae vallatae die Nervenstamme von Ganglienkugeln begleitet und stimmen hierin meine Beobachtungen mit denen $\mathrm{Szabad-}$ földy's ${ }^{1}$ ) überein. In der Papille selbst dagegen habe ich deren niemals gefunden.

Bei der weiteren Schilderung des Verlaufs der Nerven unter und in einer Papilla vallata ist es zweckmässig, markhaltige und marklose Nervenfasern besonders $z u$ betrachten. Ueber erstere orientirt man sich am besten an Schnitten durch Zungenstückchen, die in Ueberosmiumsäure erhärtet wurden. Es zeigen, was den Reichthum an markhaltigen Fasern betrifft, die Geschmackspapillen der

1) Beiträge zur Histologie der Zungenschleimhaut. Virchow's Archiv Bd. 38 1867. p. 182. 
verschiedenen Säugethiere sehr grosse Verschiedenheiten. So habe ich z. B. beim Schwein nur sehr wenige derselben am Grund der Papille gefunden; zahlreicher sind sie bei Hund und Mensch. Am reichlichsten damit versehen sind die Papillae vallatae des Schafes (vergl. Fig. 1). Hier erkennt man unter jeder derselben und zwar noch unter dem Niveau des Wallgrabengrundes einen reichlichen Plexus markhaltiger Nervenfasern, die sich hier in den verschiedensten Richtungen und in der mannigfachsten Weise durchschlingen und denen des Glossopharyngeus-Stammes, was ihre Dicke anbetrifft, vollständig gleichen. Sie sind ebenfalls meistens sehr dünn; nur wenige dickere bis $0,007 \mathrm{~mm}$. im Durchmesser kommen zur Beobachtung. Begreiflich ist es, dass man an Schnittpräparaten zahlreiche Bruchstucke markhaltiger Nervenfasern erhält, da dieselben $\mathrm{ja}$ in den verschiedensten Ebenen verlaufen.

Aus dem eben beschriebenen Plexus entwickeit sich nun ein ansehnliches Bündel dunkelcontourirter Nervenfasern, welche nach der Oberfläche der betreffenden Papille zu divergirend ausstrahlen. Demgemäss steigen einige derselben direct zur Oberfläche in die Höhe, wieder andere wenden sich mehr seitlich, jedoch alle unter mannigfachen Schlängelungen, so dass nur selten eine Faser in ihrem ganzen Verlauf in derselben Schnittebene bleibt. Oft sieht man auch schlingenförmige Umbiegungen einzelner Fasern, ohne dass jedoch irgendwo eine besondere Beziehung markhaltiger Nervenfasern zu den Schmeckbechern $z u$ constatiren wäre. Ganz ähnlich verhalten sich in dieser Beziehung Mensch und Hund. Wie man auf Fig. 1 ferner sieht, treten einige wenige markhaltige Fasern auch seitlich dicht neben dem Grunde des Wallgrabens in die Papille ein, deren Verbreitungsbezirk aber nicht über die Nachbarschaft der Seitenwände hinauszugehen scheint.

Viel reicher sind die Papillae vallatae an marklosen Fasern, und zwar finden sich die meisten derselben bei den Thieren, die am meisten markhaltige zeigen, z. B. beim Schwein. Während wir nun letztere in den allerverschiedensten Richtungen eine Papilla vallata durchkreuzen sehen, ohne dass gerade zu der Schmeckbecher-Region hin auffallend viele derselben verlaufen, zeigen die eintretenden Stämme markloser Nervenfasern, soviel man an Holzessig-Präparaten erkennen kann, ein etwas anderes Verhalten. Für das Studium ihres Verlaufs eignen sich besonders Schnitte durch Papillen vom Schwein, die vorher zwei Tage in gereinigtem Holzessig gelegen 
hatten. Man erkennt dann, dass der dicke eintretende Nervenstamm sich alsbald in zwei grössere Bündel theilt, die nach den beiden Seiten des Schnittes auseinander weichen und zur Region der Schmeckbecher hinstreben (Fig. 6), während nur wenige Faserzuge zur freien Oberfläche der Papille hin zu verfolgen sind. Im Ganzen betrachtet findet also in einer umwallten Papille vom Schwein ein wirtelförmiges Ausstrahlen zahlreicher Nervenbündel von einem Puncte aus Statt, der unter der Mitte des Geschmackswärzchens liegt. Dass in der That dieselben zur Region der Schmeckbecher verlaufen, erkennt man an denselben Präparaten bei stärkerer, etwa 350facher Vergrösserung sehr deutlich (Fig. 21). Es verlieren sich hier die betreffenden Faserzüge in einem auch nach langer Holzessig-Einwirkung immer noch dunkel erscheinendem sehr kernreichen Stratum, auf dem die Schmeckbecher unmittelbar aufsitzen. Wo diese fehlen, fehlt auch das dunkle Stratum, so dass man eine enge Beziehung beider Bildungen zu einander mit Sicherheit annehmen kann. Hier haben wir offenbar die letzten Enden der Geschmacksnerven zu suchen; hier beginnen aber auch die Schwierigkeiten, die sich einer weiteren Verfolgung der feinen Nervenfasern uns in den Weg stellen. Dass Holzessig, Chlorgold und Ueberosmiumsäure uns im Stich lassen, habe ich schon erwähnt. Letztere ermöglicht es nur noch einzelne markhaltige $\mathrm{Fa}$ seru von grosser Feinheit $(0,0018$ bis $0,0027 \mathrm{~mm}$.) dicht unter den Schmeckbechern wahrzunehmen, die hier in den mannigfachsten Richtungen und Krümmungen verlaufen. Deren sind aber im Ganzen nur wenige und von mir bis jetzt nur beim Schaf mit Sicherheit constatirt, so dass wir offenbar in ihnen allein nicht die Fasern des Glossopharyngeus suchen können, welche die Geschmacksempfindungen vermitteln. Vielmehr scheinen sich die marklosen Remak'schen Fasern dabei inniger zu betheiligen, wie aus folgenden der Schwierigkeit des Gegenstands entsprechend allerdings luickenhaften Beobachtungen hervorgeht.

Zerzupft man das oben beschriebene kernreiche Stratum nach mehrtägiger Maceration in Lösungen von Chromsäure $(1 / 50 \%$ oder) Kali bichromicum $(1 / 2$ bis $1 \%$ ) möglichst fein, was hier allerdings nur mit grosser Geduld auszufuhren ist, so findet man ausser elastischen Fasern und feinen Bindegewebsfibrillen noch eigenthumliche mit elliptischen Kernen besetzte Faserzüge, die in ihrem weiteren Verlauf sich bald wiederholt dichotomisch theilen und so in immer 
feinere Bündel spalten. Betrachtet man eins der dünneren Bündel (Fig. 19), so erkennt man zunächst mehrere, im abgebildeten Falle z. B. zwei, secundäre Bündel. Letztere machen an gewissen Stellen bei oberflächlicher Betrachtung ganz den Eindruck von markhaltigen Fasern mit Kernen in der Scheide. Gegen diese Deutung spricht jedoch schon der Umstand, dass an anderen Stellen ihres Verlaufs das vermeintliche Mark nur noch undeutlich zu erkennen ist, während im Innern der Faser fibrilläre Streifung auftritt. Am abgerissenen Ende sieht man meist mehrere feine Fibrillen hervorstehen. Diese eben beschriebenen Fasern sind nun höchst wahrscheinlich identisch mit den von Lovén beschriebenen und in Fig. $8 \mathrm{a}$ und $\mathrm{b}$ abgebildeten kernreichen markhaltigen Nervenfasern, die er mittelst derselben Methode, wie ich, aus dem zähen Stroma isolirte. Lovén gibt an, dass das Mark ziemlich plötzlich verschwinde und der Axencylinder frei werde, den er sich einmal dichotomisch theilen sah. Offenbar entsprechen die Figuren Lovén's ziemlich genau meiner Fig. 19. Ich stimme nur darin Lo vén rollkommen bei, dass die fraglichen Gebilde Nervenfasern sind, kann sie aber nicht fúr markhaltig halten. Dagegen spricht einmal der stellenweise Mangel der vermeintlichen Markscheide, ohne dass man an diesen Stellen eine Verschmälerung der Faser bemerkt, vor Allem aber ihr Verhalten gegen gewisse Reagentien. Setzt man nämlich einen Tropfen Essigsäure zum Präparat, so sieht man die dunkeln Contouren der Faser verblassen, während das Innere derselben in seinem Aussehen sich kaum verändert. Es werden jetzt die Fibrillen im ganzen Verlauf des Faserstranges deutlich, auch da, wo man fruher keine erkannte (Fig. 20). Von besonders abgegrenzten Nervenfasern ist nichts mehr zu sehen. Man erkennt einen dickeren Fibrillenstamm, der sich peripherisch in immer feinere Fibrillenästchen spaltet. An letzteren sitzen die nach Essigsäurezusatz deutlicher gewordenen elliptischen Kerne in reichlicher Menge. Stellenweise sieht man aber (Fig. 20 a) dem Fibrillenstrange eine feinkörnige Masse mit ganz ähnlichen Kernen anliegen; und dies gibt uns den Schlussel fur die Auffassung der fraglichen Bildungen. Wir erkennen in der feinkörnigen Masse den Rest einer Scheide, die durch Essigsäurezusatz verblasst ist. Diese Scheide umgibt an unveränderten Präparaten die einzelnen Bündel und scheidet stellenweise secundäre Bundelshen ab; zugleich birgt sie die Kerne. Durch Faltungen und Runzelungen der Scheide 
in Folge der Einwirkung der angewandten Reagentien kommt dann ein Aussehen zu Stande, wie es manche markhaltige Fasern zeigen. Zum Ueberfluss will ich noch hinzufugen, dass die wirklichen feinen markhaltigen Fasern dicht unter den Schmeckbechern keine Kerne erkennen lassen. Es bleibt also, da das Verhalten gegen Essigsäure die Annahme, dass man es im vorliegenden Falle mit Bindegewebsbündeln zu thun habe, ausschliesst, an elastısche Fasern aber nach Allem nicht zu denken ist, nur noch die einzige Möglichkeit, dass hier Bundel feiner Nervenfibrllen vorliegen, etwa der Art, wie sie M. Schultze aus der Geruchsschleimhaut der verschiedensten Wirbelthiere beschreibt.

Welches ist nun aber das Endschicksal dieser in eine kernreiche Scheide eingeschlossenen Nervenfibrillen? Die Entscheidung dieser Frage bietet begreiflicher Weise sehr grosse Schwierigkeiten dar, und ist es mir trotz der grössten darauf verwandten Mihe nicht gelungen, zu einem befriedigenden Resultat zu gelangen. Was sich darủber sicher aussagen lässt, ist Folgendes: Beim Zerzupfen des kernreichen festen Stratums unmittelbar unter den Schmeckbechern erhält man zahlreiche feine blasse Fasern, die den Auslaufern der Geschmackszellen sehr ähnlich sehen und gegen Essigsäure resistent sind. Von elastischen Fasern unterscheiden sie sich sofort durch ihre blassen Contouren. Sie sind also für feinste Nervenfasern zu halten und verlaufen hier in den allerverschiedensten Richtungen, einen subepithelialen Plexus bildend. Höchst wahrscheinlich stammen sie grösstentheils von den oben beschriebenen blassen Nervenfaserzugen $a b$, deren Schicksal wir direct nicht weiter verfolgen konnten. Wenigstens gleichen sie den Fibrillen derselben vollstandig.

Fúr die Frage nach der schliesslichen Endigung der beschriebenen Fäserchen wird nun aber eine Beobachtung entscheidend, der zufolge man annehmen muss, dass dieselben die Grenze zwischen Bindegewebe und Epithel uberschreiten, also in letzteres eindringen. Man sieht nämlich zuweilen nach dem Abpinseln des Epithels in dunnen Chromsäurelösungen macerırter Präparate ganz ähnliche Fáserchen frei über die Grenzfläche des Bindegewebes hervorstehen. Dieselben sehen wie abgerissen aus und gleichen sehr den centralen Ausläufern der Geschmackszellen. Da ich nun zwischen den Elementen der Schmeckbecher nie Faserchen wahrgenommen habe, die etwa als Nervenfibrillen zu deuten wären, so halte ich es nach Allem für das Wahrscheinlichste, dass jene feinen Nervenfasern direct mit 
den centralen Fortsätzen der Geschmackszellen in Zusammenhang stehen, obwohl ich nicht so glucklich gewesen bin, eine solche Verbindung zu beobachten.

Von den eigenthumlıchen birnförmigen Gebilden, in welche nach Sza badföld ${ }^{1}$ ) die Nervenfasern der Papillae vallatae auslaufen sollen und die er desshalb für die peripherischen Endorgane der Geschmacksnerven hält, habe ich, obgleich ich die verschiedensten Methoden in Anwendung gebracht habe, nie etwas gesehen. Auch Terminalkörperchen, wie sie W. Krause ${ }^{2}$ ), als Endigungen der sensibeln Nervenfasern in den umwallten Papillen des Menschen beschreibt, sind mir beim Schaf und Schwein, die ich besonders genau auf diese Verhältnisse untersucht habe, nicht zu Gesicht gekommen.

Sehen wir uns nun, nachdem wir die Geschmacksorgane der Sáugethiere kennen gelernt haben, nach analogen Bildungen in der Wirbelthierreihe um, so fält zunächst die grosse Uebereinstimmung der Schmeckbecher mit den von Ley dig ${ }^{3}$ ) entdeckten ,becherförmigen Organen" der Fische auf, uber deren wahre Natur uns Fr. E. $\mathrm{Schulz} \mathrm{e}^{4}$ ) aufgeklärt hat. Er erklärte dieselben, gestützt auf seine histologischen Untersuchungen, zuerst mit Entschiedenheit für Geschmacksorgane. Nach der Auffindung ganz ähnlicher Gebilde an der Stelle der Zunge der Saugethiere und des Menschen, welche von allen Physiologen als schmeckend anerkannt ist, kann wohl über die Bedeutung der ,becherformigen Organe“ der Fische auch nicht mehr der leiseste Zweifel herrschen. Sogar die frei hervorragenden Stiftchen der Geschmackszellen finden bei den Fischen ihre Analoga, wie eine Beobachtung von F. E. Schulzes) lehrt.

Ganz anders scheinen sich auf den ersten Blick die Geschmacksorgane der Frösche zu verhalten. Meiner Meinung nach verschwindet aber die grosse Verschiedenheit, wenn man das ganze Nervenepithel einer Papilla fungiformis als einem Schmeckbecher gleichwerthig ansieht. Auch die zelligen Elemente der letzteren haben in

1) 1. c. p. 184

2) Ueber die Nervenendigung in den Papillae circumvallatae der menschlichen Zungé. Gottinger Nachrichten, 1863.

3) Ueber dì Haut einiger Süsswasserfische. Zeitschrift für wissenschaftliche Zoologie. Band III. p 3.

4) Ueber die becherförmigen Organe der Fische. Zeitschrift für wissenschaftliche Zoologie. Band XII. p. 218.

5) Epithel- und Druisen-Zellen. Dieses Archiv. Bd. III. p. 153. 
den modificirten Epithelialzellen und Geschmackszellen A. Key's ihre Analoga. Freilich scheint nach den kurzlich publicirten interessanten Beobachtungen En gelman n's ${ }^{1}$ ) uber denselben Gegenstand das Nervenepithel der Pap. fungiformes der Froschzunge noch complicirter zusammengesetzt zu sein, und lassst sich desshalb eine genaue Vergleichung der Bestandtheile desselben mit denen eines Schmeckbechers für jetzt nicht durchfuhren.

Bei den Repitilien und Vogeln endlıch sind die Geschmacksorgane noch gänzlich unbelannt. Die wenigen Untersuchungen, die ich bis jetzt an der Zunge und Pharynxschleimhaut einiger Vögel (Ente, Huhn, Taube) anstellen konnte, haben noch zu keinem positiven Resultat gefuhrt. Wahrscheinlich sind gerade die Zungen der kơrnerfressenden Vögel die ungûnstigsten Objecte zur Entscheidung.

Schliesslich habe ich noch dankbar anzuerkennen, dass ich auch bei dieser Arbeit, die im anatomischen Institut zu Bonn begonnen wurde, mich vielfach des freundlichsten Rathes des Herrn Professor Max Schultze zu erfreuen hatte.

Nachtrag. Vorstehende Zeilen waren schon niedergeschrieben, als mir die deutsche Uebersetzung der Arbeit Lovén's im vorigen Hefte dieses Archivs bekannt wurde. Verfasser hat ihr einige Zeilen als Nachschrift hinzugefugt, in welchen er einige neue Beobachtungen uber die Verbreitung der Geschmacksknospen auf der Zunge anfuhrt. Auch Lovén fand deren, wie ich, vereinzelt an der Wallseite des Ringthals, so dass uber diesen Punct wohl kein Zweifel herrschen kann. Es sind ja ùberdies die Bedingungen hier ebenso günstig, als an der Papillenseite. Anders steht es mit den Angaben Lovén's, er habe auch auf der freien Oberfläche der Papillae fung if ormes Geschmacksknospen gefunden. Es wurde dann die versteckte Lage derselben durchaus nicht charakteristisch sein, und man wurde nicht einsehen, wesshalb nicht auch auf der freien Obertäche der umwallten Papillen Schmeckbecher vorkommen sollten. Hier hat aber auch Lovén deren nicht beobachtet. Meine fruberen Untersuchungen an Schaf und Rind haben, wie ich oben ausfuhrte, ergeben, dass an den Papillae fungiformes

1) Ueber die Endigungsweise der Geschmacksnerven des Frosches. Medicinisches Centralblatt. 181,7 . Nr 50. p. 785 und Zeitschrift fur wissenschaftluche Zoologie. 1868. 
dieser Thiere keine Schmeckbecher vorkommen. Lovén empfiehlt jetzt, um sich davon zu überzeugen, die pilzförmigen Papillen des Kaninchens und der Ratte. Mir stand augenblicklich nur ersteres zu Gebote. Die wenigen Untersuchungen, die mir die Kürze der Zeit noch gestattete vorzunehmen, ergaben, dass allerdings eine Flächenansicht, der freien Oberfläche der genannten Papillen ganz ähnlich aussieht, wie die der Schmeckbecher-Region der Papillae vallatae. Man erkennt grössere Kreise in einem Maschenwerk gewöhnlich angeordneten Epithels. Allein von der Existenz von Oeffnungen im Epithel konnte ich mich nicht überzeugen, wohl aber von der Anwesenheit langer schmaler secundärer Papillen, die das sehr dicke Epithel bis dicht unter die Oberfläche durchsetzten. Sodann gelang es mir nicht, Schmeckbecher durch die oben genannten Methoden von dieser Stelle zu isoliren. Dies Verfahren ist aber meiner Ansicht nach immer zur Controle anzuwenden, da Oberflächenansichten zur Entscheidung der Frage nicht genügen und $z$ war aus folgenden Gründen. Um die Spitze der secundären Papillen herum ist das Epithel concentrisch angeordnet. Stehen nun zahlreiche derselben dicht neben einander, so wird eine Flächenansicht ebenfalls Kreise zeigen, welche dicht neben einander in einem Maschenwerk gewöhnlich angeordneten Epithels liegen. So scheinen mir denn in der That sich die Papillae fungiformes des Kaninchens zu verhalten. Doch gestehe ich gern zu, dass meine Beobachtungen noch nicht genügend zahlreich waren, um mich mit voller Sicherheit negirend aussprechen zu können. Von acinösen Drüschen, die sonst immer in der Umgebung der Schmeckbecher sich finden, sah ich an jenen Papillen nichts. Uebrigens hätte das Vorkommen von Schmeckbechern auf den Papillae fungiformes an sich nichts Auffälliges, da ja nach $R$ ü dinger der Glossopharyngeus beim Menschen makroskopisch ziemlich weit über das Gebiet der Papillae vallatae hinaus nach vorn zu verfolgen ist. Das Auffallende in L ovén's Angaben liegt vielmehr darin, dass die Geschmacksknospen in einzelnen Fällen an der frei en Oberfäche der Papillen vorkommen, wo sie offenbar den verschiedensten mechanischen Insulten ausgesetzt sind, während für den grössten Theil derselben als Gesetz gilt, dass sie immer an möglichst geschützten Stellen der Zungenschleimhaut gefunden werden. 


\section{Erklärung der Abbildungen \\ auf Tafel XII und XIII.}

Fig. 1, Fig. 3 bis 9 und Fig. 22 sind bei der Vergrösserung System C Ocular II eines Zeis'schen Mikroskopes, Fig: 2 und 21 bei D Ocular II, Fig. 10 bis 20 bei System F Ocular II desselben Mikroskopes gezeichnet.

Fig. 1. Senkrechter Schnitt durch eine Papilla vallata vom Schaf nach Erhärtung in Ueberosmiumsäure von $\left.1^{\circ}\right|_{4}$. Man erkennt an der dem Walle zugekehrten Seite der Papille jederseits sieben Schmeckbecher, welche die ganze Dicke des Epithels vom bindegewebigen Stroma an bis zu der den Wallgraben begrenzenden Obertläche durchsetzen. In den Grund des Ringthales münden links zwei, rechts ein Ausführungsgang acinöser Drüschen. Am Grunde der Papilie ein Plexus markhaltiger Nervenfasern, aus welchem sich mehrere derselben bis in die Näbe des Epithels erheben und hier theilweise schlingenförmig umbiegen. Seitlich von dem Plexus treten noch einzelne markhaltige Fasern ein.

Fig. 2 A. Schnitt durch das Epithel einer schwarz pigmentirten Papilla fungiformis vom Schaf mit verästelten Pigmentzellen in den untersten Schichten des Epithels zwischen den langen spitzen secundären Papillen. Mehrere lange dünne Pigmentfäden dringen in gerader Richtung nach aussen weit durch das Epithel hindurch. Zeichnung des Herrn Prof. M. Schultze.

Fig. 2 B. Schnitt durch das Epithel der freien Oberfläche einer schwarz pigmentirten Papilla vallata vom Schaf. Ueberosmiumsäure-Präparat. Zeigt im Wesentlichen dieselben Verhältnisse wie $2 \mathrm{~A}$.

Fig. 3. Schnitt durch eine Papilla vallata des Menschen. Ueberosmiumsäure-Präparat. Die Papillc ist etwas nach links überhängend. Auch im Epithel des Walles stecken jederseits drei Schmeckbecher. a Ausfübrungsgang einer acinösen Drüse.

Fig. 4. Schnitt durch eine umwallte Papille des Menschen. Ringwall rechts nicht entwickelt. Es fehlen desshalb hier auch die Schmeckbecher im Epitbel, während links, wo der Wallgraben sehr tief und schmal ist, deren vorkommen. Auf der Mitte der Papille öffnet sich der Ausführungsgang einer acinösen Drüse (b). a Ausführungsgänge solcher Drüschen. Ueberosmiumsăure-Präparat.

Fig. 5. Schnitt durch eine Papilla vallata vom Reh. UeberosmiumsäurePräparat. Auf einer vollständig umwallten Papille sitat eine kleinere pilzförmige auf, letztere ohne Schmeckbecher. a wie vorhin.

Fig. 6. Schnitt durch eine Papilla vallata des Schweines. Dieselbe ist sehr breit, mit dellenförmig eingedrückter Oberfläche. a wie vorhin; b eintretender Nervenstamm, der sich in zwei divergirende Aeste theilt. Ueberosmiumsäure-Präparat. 
Fig. 7. Schnitt durch eine umwallte Papille des Ochsen. Stark entwickelter überhängender Ringwall mit sehv verdicktem Epithel. a wie vorhin. Ueberosmiumsäure-Präparat.

Fig. 8. Flächenschnitt durch eine Papilla vallata rom Schaf. Man erkennt ringsum im Epithel Schmeckbecher.

Fig. 9. Oberflächenansicht eines Theils der Seitenwand einer Papilla vallata vom Schwein, bei tiefer Einstellung. Man erkennt in dem dunkeln Maschenwerk des gewöhnlichen Epithels helle Kreise. Dieselven entsprechen den optischen Querschnitten der Schmeokbecher und sind beim Schwein unregelmässig gestellt und von verschiedener Grösse. Links sind im Centrum von fünf derselben kleine Kreise eingezeichnet, die aber erst bei Einstellung auf die äusserste Oberfläche deutlich sind; sie entsprechen Löchern im Epithel. Präparat nach Maceration in Kali bichromicum von $2 \%$ gewonnen.

Fig. 10 a. Aeusserste Oberfläche der Seitenwand einer umwallten Papille vom Schwein, nach Maceration in Jodserum abgehoben. Man erkennt drei kleine helle Kreise als Ausdruck dreier Oeffnungen im Epithel, die beim Schwein sehr klein sind; dieselben stehen je auf einer sanften uhrglasförmigen Erhabenheit. An der Bildung des Epithelloches betheiligen sich meist drei Zellen. b. Zwei Zellen bilden ein solches Loch.

Fig. 11. Durch Ueberosminmsäure von $1 \%$ isolirter Schmeckbecher vom Schaf. Aus der geschlossenen Knospe sehen drei kleine Stifte hervor. Ausserdem erkennt man viele elliptische, mit ihrem Längsdurchmesser parallel dem Längsdurchmesser des Schmeckbechers gestellte Kerne ohne deutliche Zellengrenzen. Die dem Bindegewebe aufsitzende Basis ist ausgefasert.

Fig. 12. Peripherisches Ende eines anderen Schmeckbechers rom Schaf (Ueberosmiumsäure $1 \frac{\mathrm{g}}{\mathrm{g}}$ ). Drei lange Stiftchen von ungleicher Grösse ragen daraus hervor.

Fig. 13. Ebenso von einem anderen Schmeckbecher des Schafes. An der Spitze ein Härchenkranz; aus der Tiefe des Schmeckbechers sieht man zwei Stiftchen unter dem Härchenkranze hervorragen. Ueberosmiumsäure $1 \frac{g}{6}$.

Fig. 14. Geöffnete Geschmacksknospe des Schafes nach Behandlung mit Kali bichromicum von $2 \%$. Die Gcschmackszellen sind herausgefallen. Man sieht einen von Deckzellen gebildeten offenen Kelch und am Grunde drei Basalzellen.

Fig. 15. a und b Deckzellen eines Schmeckbechers vom Schaf mit unverästelten centralen Fortsätzen. c bis h Geschmackszellen des Schafes, sämmtlich mit dickerem peripherischen und dünnem meist unregelmässig oder gar nicht varikösen centralen Fortsatz. In c zeigt die linke Zelle einen centralen Fortsatz mit regelmässigen Varicositäten. f Stiftchenzelle: der allmählig sich verschmälernde peripherische Fortsatz geht in ein dünnes hellglänzendes Stiftchen über. d Stäbchenzelle: der peripherische Fortsatz ist durch ein überall gleich breites Stäbchen ersetzt. Die anderen Zellen sind Stiftchenzellen, welche durch das Reagens (Kali bichr. 2o) mehr oder weniger verändert sind.

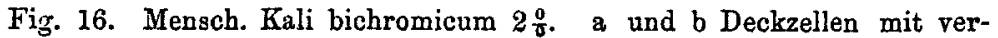
ästeltem centralen Fortsatz. c bis. f Geschmackszellen. d Stäbchenzelle mit 
Kernkörperchen. c, e und f Stiftehenzellen; das Stiftchen aber nur bei e erhalten. Die centralen Enden des dem Bundegewebe zugekehrten Ausläufers meist knopfförmig oder knollig.

Fig 17. Schaf. Kali bıchromicum $2 \&$ a und b kleine Bürstenzellen des Seiterwandepithels der Papllae vallatae. c und d Basalzellen mit ausgefasertem peripherischen Ende e Oberftacbenzelle einer umwallten Papille nit bisquitformigem Nucleus.

Fig. 18. Oberflachenzellen der Seitenwand der Papillae vallatae vum Kalb Kali bichromicum $2 \frac{8}{v}$ a Zelle mit tiefem kreisförmigen Ausschnitt entsprechend einer Oeffnung im Epithel. b Lochzelle. c etwas tiefer gelegene Epithelzelle mit Ausschnitt an der Seite, wo sie an ennen Schmeckbecher grenzte.

Fig. 19. Zwei marklose Nervenfasern aus dem Bindegewebe unmittelbar unter den Schmeckbechern vom Schaf (Kali bichromicum ${ }^{2} / 2 \frac{0}{0}$ ). Aus den abgerissenen Enden schauen einzelne Fibrillen hervor.

Fig. 20. Ein Buindel eben solcher nuarkloser Fasern vom Schaf nach Behąndlung mit Essigsäure. Die Grenzen der einzelnen Nerrenfasern sınd nicht mehr zu erkennen; die fibrillare Structur ist deutlicher geworden Zahlreiche Kerne liegen dem Bundel an. Bei a Scheidenrest mit vielen Kernen.

Fig. 21. Schnitt durch die Schmeckbecher-Region einer umwallten Papille des Schweines. Holzessig-Praparat Man sieht ein Bündel Nervenfasern zu der Gegend der Schmeckbecher ziehen und sich hier in einem dunkeln kernreichen Stratum unmittelbar unter den Geschmacksknospen verlieren. a Ausführungsgang einer Drüse.

Fig. :2. Oberflächenansicht eines Stücks der durch Maceration in Kali bichroinicum von $2 \frac{\Omega}{0}$ abgehobenen Epithelialdecke einer Paplla vallata vom Schaf. a Schmeckbecbergegend. Man erkennt zahlreiche grössere belle Kreıse, die optischen Querschnitte der Schmeckbecher und innerhalb eines jeden der"selben einen kleinen Kreis, entsprechend einem Loch im Epithel. b Epithel der freien Obertáche der Papille ohne Schmeckbecher. 